\title{
Synthetic circuits, devices and modules
}

\author{
Hong Zhang, Taijiao Jiang ${ }^{\bowtie}$ \\ National Laboratory of Biomacromolecules, Institute of Biophysics, Chinese Academy of Sciences, Beijing 100101, China \\ $\triangle$ Correspondence: taijiao@moon.ibp.ac.cn \\ Received November 4, 2010 Accepted November 9, 2010
}

\begin{abstract}
The aim of synthetic biology is to design artificial biological systems for novel applications. From an engineering perspective, construction of biological systems of defined functionality in a hierarchical way is fundamental to this emerging field. Here, we highlight some current advances on design of several basic building blocks in synthetic biology including the artificial gene control elements, synthetic circuits and their assemblies into devices and modules. Such engineered basic building blocks largely expand the synthetic toolbox and contribute to our understanding of the underlying design principles of living cells.
\end{abstract}

KEYWORDS synthetic biology, genetic circuit, synthetic device, module

\section{INTRODUCTION}

"What I cannot create, I do not understand," as said by the physicist Richard Feynman, synthetic biology has become an important tool not only to uncover the design principles of natural biologic systems, but also to explore novel biologic functions and systems not found in nature. Currently, synthetic biology is heading for two main directions. In the first direction, researchers endeavor to strip genomes down to their essential parts for construction of the minimal genomes. Several synthetic genomes have been successfully established including bacteriophage (Smith et al., 2003; Chan et al., 2005), virus (Cello et al., 2002), the smallest known genome of a free-living organism Mycoplasma genitalium (Gibson et al., 2008) and even Escherichia. coli (Pósfai et al., 2006). Recently, Craig Venter and his colleagues assembled and modified a synthesized genome into a DNA-free bacterial shell to make a self-replicating Mycoplasma mycoides (Gibson et al., 2010). Through synthesis of simple genomes, we could have an unprecedented opportunity to learn about what gene elements are basic and fundamental for life and how they are assembled into life forms.

In the other direction, novel gene elements have been identified, redesigned or designed, which are further combined to build artificial biologic systems with specified functions. From an engineering perspective, the design of a biologic system first requires the appropriate decomposition of the design task into gene elements and their basic combinations capable of operation in forms of circuits, devices and modules, and then employs a bottom-up approach to further assemble them into more complex systems in a hierarchical way. Ten years since the first two synthetic elements were introduced into this field (Elowitz and Leibler, 2000; Gardner et al., 2000), synthetic biologists have made much progress to engineer a wide range of artificial gene parts to expand their functionalities and applications. In this review, we focus on a brief introduction of synthetic circuits, devices and modules by highlighting some current achievements. We state that the materials cited in this review only represent part of the current achievements in the related area.

\section{ARTIFICIAL GENE CONTROL ELEMENTS}

Due to current extensive studies on characterization of DNA and RNA sequence segments as specific gene functional and regulatory elements including promoters, cis-transcriptional elements, riboswitches, small interfering RNAs and microRNAs, design of gene regulatory circuits offers the most successful examples for test of a hypothesis in synthetic biology.

Transcriptional motif-based synthetic elements

An efficient and general way to regulate cellular functions is to regulate gene expression. Previous studies have identified the molecular mechanisms underlying gene transcription regulation, which involve the specific interactions between 
protein factors and DNA elements. The protein factors can be functionally classified as transcription factors, activators and repressors. Given that specific DNA-protein recognition can effectively turn on/off gene expression, design of specific protein-DNA interactions has been an important means to regulate cellular activities. The zinc finger proteins are among the most studied DNA-binding proteins, which typically contain many zinc binding motifs in tandem. The zinc finger protein can recognize a wide variety of DNA sequences for different functionality, making it an attractive framework to design novel gene expression regulators. Researchers have utilized different design strategies to create novel zinc finger peptides by re-engineering the $\mathrm{C}(2) \mathrm{H}(2)$-type zinc finger motif, the (His)4-type zinc finger protein and the AT-recognizing zinc finger protein (Dhanasekaran et al., 2006; Nomura and Sugiura, 2007; Negi et al., 2008; Sera, 2010). Some new artificial zinc finger proteins have novel DNA-binding properties or even nuclease and catalytic functions. These zinc finger protein variants provide powerful molecular tools to modulate endogenous gene expression and regulate gene function.

\section{Synthetic RNA elements for post-transcriptional regulation}

Although gene transcriptional regulation has gained much attention in synthetic biology, the recent advances in ribozymes, riboswitches, interfering RNAs and other regulatory RNAs have offered new gene elements to control gene expressions at post-transcriptional level. The ability of ribozymes to recognize a well defined tertiary structure and cut specific RNA molecules makes them exciting candidates for synthetic biologists. Yen et al. effectively designed an RNA-based gene regulation system for mammalian cells via the incorporation of sequences encoding self-cleaving RNA motifs into the transcriptional unit of a gene to inhibit its expression (Yen et al., 2004). Researchers in Dr. Altman's lab developed novel methodologies for inhibition of target RNAs based on the cleavage activity of ribonuclease $P(R N a s e P)$. RNase P-mediated cleavage of target RNAs can be directed by external guide sequences (EGSs) (Lundblad et al., 2008; Shen et al., 2009).

A riboswitch is part of an mRNA molecule (usually located within the $5^{\prime}$-UTRs of mRNA) that can bind small molecules like metabolites. Upon binding, the riboswitch can affect the conformation of its adjacent mRNA, resulting in either the premature termination of transcription, inhibition of translation initiation, or mRNA self-cleavage (Winkler, 2005). Once discovered in nature, riboswitches have been quickly applied to control gene expression (Desai and Gallivan, 2004; Suess et al., 2004). Recently Dixon et al. designed riboswitches that are selective for synthetic "nonnatural" small molecules rather than the natural intracellular ligands (Dixon et al., 2010). These riboswitches with orthogonal selectivity provides effective gene expression regulators both in prokaryotes and eukaryotes. Design of riboswitches that bind protein ligands is another interesting direction. Saito et al. constructed an L7Ae-kink-turn RNA-protein (RNP) switch, in which the small molecule-RNA interaction was replaced by protein-RNA interaction (Saito et al., 2010). The design of riboswitch to be controlled by protein ligand could greatly extend its applications in cellular context.

Furthermore, the elucidation of functional roles of small interfering RNAs and microRNAs has identified them as potent gene post-transcriptional regulators. Due to their effectiveness in gene silencing in an array of organisms, small synthetic interfering RNAs (Kim et al., 2005; Siolas et al., 2005; Beisel et al., 2008) and microRNAs (Dickins et al., 2005; Suryawanshi et al., 2010) have been used to control gene expression.

Compared to protein-based synthetic circuits, ribozymes, riboswitches and small interfering RNAs are relatively easy to engineer and manipulate, offering an attractive strategy in modulating or reprogramming cellular pathways (Mulhbacher et al., 2010).

\section{Synthetic proteins}

Under cellular circumstance, specific protein-protein interactions constitute the basic events of cell signaling. How to design specific molecular interactions is critical to understand and modulate cell physiology and cellular activities. Many proteins in nature usually consist of domains organized in a cassette-like fashion. These domains either mediate molecular interactions or have other specific functions.

Sequence analyses have suggested that domain recombination is a major driving force for generation of novel protein functions. There have been an increasing number of successes in acquiring new protein functions by domain rearrangements and combinations. Therefore, design of proteins based on domain combinations can provide an effective means to generate new functions and further endow the cell with new phenotypes. By using small autonomously folded A domains as building blocks, Silverman et al. created a class of multi-domain binding proteins with improved affinity and specificity, called Avimers (Silverman et al., 2005). In some cases, creation of multi-domain proteins is not just to bring together individual domains because dramatic changes may occur at their newly formed interfaces. Huang et al. found that the optimization of the interface between PDZ and FN3 domains by combinatorial library selection of FN3 loops using phage display could create new binding interface that contributes to a dramatic increase of binding affinity and specificity (Huang et al., 2008).

Design of scaffold proteins using domain combinations is another way to create new synthetic cellular circuits. Scaffold proteins are molecules that bind multiple signaling components and promote their communication or interaction with 
each other. Synthetic protein scaffolds can redirect the flow of cellular signals. In S. cerevisiae, Ste5p is a well-characterized scaffold protein that tethers multiple protein kinases in the mitogen-activated protein (MAP) kinase cascade required for mating and Pbs2 is involved in osmo-regulatory pathway. By creating a chimera scaffold with Ste5 and Pbs2, Park et al. have redesigned yeast mitogen-activated protein kinase (MAPK) pathways that were able to generate mating responses when stimulated with high salt (Park et al., 2003).

In signaling networks, domains generally carry out two functions: regulation and catalysis. Theoretically recombination of such different functional domains could generate novel signal circuits and new phenotypes. To test this idea, by constructing a library of 66 chimeric domain recombinants from 11 proteins of different functions in the yeast mating pathway which were specifically split in a manner that separated regulatory and catalytic domains, Peisajovich et al. found that domain recombinations resulted in greater diversity in pathway response dynamics and led to changes in mating phenotype (Peisajovich et al., 2010).

\section{GENETIC CIRCUITS AND DEVICES}

Synthetic biology aims to merge engineering approaches with biology. The integration of the above synthetic elements to genetic circuits and devices can be analogous to the assembly of resistors and transistors into the electronic circuitry.

\section{Genetic switches}

The function of genetic switches enables cells to make conditional transitions between two states, generally through gene transcriptional regulation. The genetic switches based on transcription factors utilize diverse mechanisms to achieve their functionalities, which include: (1) the conformational change of transcription factors according to the environmental signals (Zhang et al., 2009), (2) translocation of transcriptional regulators (Buchler and Cross, 2009; Ghim and Almaas, 2009), (3) accession or depletion of small molecules (Sprengel and Hasan, 2007), and (4) response to several special signals, such as light, $\mathrm{pH}$ and redox potential (Pomposiello and Demple, 2001; Georgiou, 2002; Levskaya et al., 2009; Paulsen and Carroll, 2009; Tietze et al., 2009). More sophisticated genetic switches have also been engineered including bistable switches (Kim et al., 2006) and memory push-on push-off switches (Lou et al., 2010).

\section{Oscillators}

Cells exhibit a range of oscillatory behaviours such as the cell cycling and the circadian oscillation. De novo designed oscillators can be used not only to test the design principles of natural oscillation, but also to explore novel applications. To achieve transcriptional and metabolic integration characteristics of natural oscillators, Fung et al. constructed a synthetic oscillator in E. coli using glycolytic flux to generate oscillation through interconverting the signalling metabolites by two enzymes (Fung et al., 2005). Tigges et al. designed an oscillator to enable autonomous, self-sustained and tunable oscillatory gene expression in mammalian cells based on an auto-regulated transcription control circuit encoding a positive and a time-delayed negative feedback loop (Tigges et al., 2009). Using a modeled network architecture comprising linked positive and negative feedback loops, Stricker et al. engineered a fast, robust and tunable genetic oscillator, whose robustness is achieved by introduction of a time delay in the negative feedback loop (Stricker et al., 2008). The synthetic oscillators provide insights into the dynamics of natural periodic processes and foster advances in the design of networks in future gene and cell therapies.

\section{Higher-order genetic circuits and devices}

Simple genetic switches or logic gates can be combined to yield higher-order genetic circuits of given functionalities to achieve complex cellular regulation. The construction of such higher-order genetic circuits and devices has allowed the synthetic biologists to challenge more difficult works, such as output of specific signal mode (Kemmer et al., 2010), noise control (Hooshangi et al., 2005; Murphy et al., 2010), biological counters (Friedland et al., 2009) and circadian clocks (Danino et al., 2010). Given the broad applications of these genetic circuits and devices, it is crucial to develop more useful devices that operate effectively inside living cells.

\section{SYNTHETIC BIOLOGICAL MODULES}

A biological module is a compartmentalized set of genetic devices with interconnected functions that performs complex tasks. In the cell, modules are specific pathways, such as a metabolic pathway or a signal transduction pathway (Andrianantoandro et al., 2006). The most prominent examples of synthetic modules are the engineered metabolic pathways. When new biochemical reactions were introduced into natural fatty acid synthesis pathway, fatty acid metabolism can be directed towards synthesis of fuel and chemical products of interest (Steen et al., 2010). Atsumi et al. engineered a cyanobacterium, Synechococcus elongatus, to produce isobutyraldehyde and isobutanol directly from $\mathrm{CO}_{2}$ (Atsumi et al., 2009). Artificial modules can also be utilized to produce drugs and commercial compounds (Martin et al., 2003; Ro et al., 2006; Zhang et al., 2008) or to construct biosensors in medical and environmental settings (Kobayashi et al., 2004; Radhika et al., 2007). The advances from these works may eventually allow us to construct cell-based systems with any desired applications. 


\section{PERSPECTIVES}

Recent advances have demonstrated achievements in design of some genetic circuits, devices and modules, particularly those for gene expression regulation. However, these designs are mainly for proof of concepts and far from generalization and rationalization. From an engineering perspective, a more focused and targeted endeavor on the design parts list could greatly facilitate the standardization of design parts and their logics. Further work could be concentrated on systematically mapping functional motifs and more importantly, quantifying the effects of their combinations as linear signal cascade and in the context of complex connections. After systematic characterization, these motifs/domains should be further standardized in terms of sequence specification and functional performance by combining computational and experimental strategies. For some applications, a synthetic system is required to operate independently in the context of an organism, while for other applications it requires the synthetic system to be integrated with the cellular activity. A systematic modeling of the interactions within the designed system or between the designed system and natural environment is critical for these specified and desired functionalities. The integrative effect of the basic building blocks may not be a simple linear combination. Computational modeling can greatly facilitate the optimization process of designing network-based artificial devices. Recently, researchers were able to accurately model the relationship between combinations of cis-regulatory elements and the gene expression levels in different environments (Gertz and Cohen, 2009; Gertz et al., 2009; Mogno et al., 2010).These work suggest the possibility of rational design of genetic circuits with desired outcomes.

\section{ACKNOWLEDGEMENTS}

This review was adapted from a work paper we prepared for Exploratory Round Table Conference (ERTC) for Synthetic Biology between CAS of China and MPG of Germany. We thank Professors GP Zhao and C Yang of Institute of Plant Physiology and Ecology, CAS for stimulating discussions and members of Jiang' lab for comments on the manuscript. This work was supported by the National Basic Research Program of China (973 Program) (Nos. 2009CB918503 and 2006CB911002) to TJ.

\section{REFERENCES}

Andrianantoandro, E., Basu, S., Karig, D.K., and Weiss, R. (2006). Synthetic biology: new engineering rules for an emerging discipline. Mol Syst Biol 2, 2006.0028.

Atsumi, S., Higashide, W., and Liao, J.C. (2009). Direct photosynthetic recycling of carbon dioxide to isobutyraldehyde. Nat Biotechnol 27, 1177-1180.

Beisel, C.L., Bayer, T.S., Hoff, K.G., and Smolke, C.D. (2008). Modelguided design of ligand-regulated RNAi for programmable control of gene expression. Mol Syst Biol 4, 224-237.

Buchler, N.E., and Cross, F.R. (2009). Protein sequestration generates a flexible ultrasensitive response in a genetic network. Mol Syst Biol 5, 272-278.

Cello, J., Paul, A.V., and Wimmer, E. (2002). Chemical synthesis of poliovirus cDNA: generation of infectious virus in the absence of natural template. Science 297, 1016-1018.

Chan, L.Y., Kosuri, S., and Endy, D. (2005). Refactoring bacteriophage T7. Mol Syst Biol 1, 20050018.

Danino, T., Mondragón-Palomino, O., Tsimring, L., and Hasty, J. (2010). A synchronized quorum of genetic clocks. Nature 463 , 326-330.

Desai, S.K., and Gallivan, J.P. (2004). Genetic screens and selections for small molecules based on a synthetic riboswitch that activates protein translation. J Am Chem Soc 126, 13247-13254.

Dhanasekaran, M., Negi, S., and Sugiura, Y. (2006). Designer zinc finger proteins: tools for creating artificial DNA-binding functional proteins. Acc Chem Res 39, 45-52.

Dickins, R.A., Hemann, M.T., Zilfou, J.T., Simpson, D.R., Ibarra, I., Hannon, G.J., and Lowe, S.W. (2005). Probing tumor phenotypes using stable and regulated synthetic microRNA precursors. Nat Genet 37, 1289-1295.

Dixon, N., Duncan, J.N., Geerlings, T., Dunstan, M.S., McCarthy, J.E., Leys, D., and Micklefield, J. (2010). Reengineering orthogonally selective riboswitches. Proc Natl Acad Sci USA 107, 2830-2835.

Elowitz, M.B., and Leibler, S. (2000). A synthetic oscillatory network of transcriptional regulators. Nature 403, 335-338.

Friedland, A.E., Lu, T.K., Wang, X., Shi, D., Church, G., and Collins, J. J. (2009). Synthetic gene networks that count. Science 324 , 1199-1202.

Fung, E., Wong, W.W., Suen, J.K., Bulter, T., Lee, S.G., and Liao, J.C. (2005). A synthetic gene-metabolic oscillator. Nature 435, 118-122.

Gardner, T.S., Cantor, C.R., and Collins, J.J. (2000). Construction of a genetic toggle switch in Escherichia coli. Nature 403, 339-342.

Georgiou, G. (2002). How to flip the (redox) switch. Cell 111, 607-610.

Gertz, J., and Cohen, B.A. (2009). Environment-specific combinatorial cis-regulation in synthetic promoters. Mol Syst Biol 5, 244-252.

Gertz, J., Siggia, E.D., and Cohen, B.A. (2009). Analysis of combinatorial cis-regulation in synthetic and genomic promoters. Nature 457, 215-218.

Ghim, C.M., and Almaas, E. (2009). Two-component genetic switch as a synthetic module with tunable stability. Phys Rev Lett 103, 028101.

Gibson, D.G., Benders, G.A., Andrews-Pfannkoch, C., Denisova, E. A., Baden-Tillson, H., Zaveri, J., Stockwell, T.B., Brownley, A., Thomas, D.W., Algire, M.A., et al. (2008). Complete chemical synthesis, assembly, and cloning of a Mycoplasma genitalium genome. Science 319, 1215-1220.

Gibson, D.G., Glass, J.I., Lartigue, C., Noskov, V.N., Chuang, R.Y., Algire, M.A., Benders, G.A., Montague, M.G., Ma, L., Moodie, M. M., et al. (2010). Creation of a bacterial cell controlled by a chemically synthesized genome. Science $329,52-56$.

Hooshangi, S., Thiberge, S., and Weiss, R. (2005). Ultrasensitivity and noise propagation in a synthetic transcriptional cascade. Proc Natl Acad Sci U S A 102, 3581-3586.

Huang, J., Koide, A., Makabe, K., and Koide, S. (2008). Design of protein function leaps by directed domain interface evolution. Proc Natl Acad Sci U S A 105, 6578-6583.

Kemmer, C., Gitzinger, M., Daoud-El Baba, M., Djonov, V., Stelling, J., and Fussenegger, M. (2010). Self-sufficient control of urate 
homeostasis in mice by a synthetic circuit. Nat Biotechnol 28 , 355-360.

Kim, D.H., Behlke, M.A., Rose, S.D., Chang, M.S., Choi, S., and Rossi, J.J. (2005). Synthetic dsRNA Dicer substrates enhance RNAi potency and efficacy. Nat Biotechnol 23, 222-226.

Kim, J., White, K.S., and Winfree, E. (2006). Construction of an in vitro bistable circuit from synthetic transcriptional switches. Mol Syst Biol 2, 68-79.

Kobayashi, H., Kaern, M., Araki, M., Chung, K., Gardner, T.S., Cantor, C.R., and Collins, J.J. (2004). Programmable cells: interfacing natural and engineered gene networks. Proc Natl Acad Sci U S A 101, 8414-8419.

Levskaya, A., Weiner, O.D., Lim, W.A., and Voigt, C.A. (2009). Spatiotemporal control of cell signalling using a light-switchable protein interaction. Nature 461, 997-1001.

Lou, C., Liu, X., Ni, M., Huang, Y., Huang, Q., Huang, L., Jiang, L., Lu, D., Wang, M., Liu, C., et al. (2010). Synthesizing a novel genetic sequential logic circuit: a push-on push-off switch. Mol Syst Biol 6, 350-360.

Lundblad, E.W., Xiao, G., Ko, J.H., and Altman, S. (2008). Rapid selection of accessible and cleavable sites in RNA by Escherichia coli RNase $\mathrm{P}$ and random external guide sequences. Proc Natl Acad Sci U S A 105, 2354-2357.

Martin, V.J., Pitera, D.J., Withers, S.T., Newman, J.D., and Keasling, J.D. (2003). Engineering a mevalonate pathway in Escherichia coli for production of terpenoids. Nat Biotechnol 21, 796-802.

Mogno, I., Vallania, F., Mitra, R.D., and Cohen, B.A. (2010). TATA is a modular component of synthetic promoters. Genome Res 20, 1391-1397.

Mulhbacher, J., St-Pierre, P., and Lafontaine, D.A. (2010). Therapeutic applications of ribozymes and riboswitches. Curr Opin Pharmacol 10, 551-556.

Murphy, K.F., Adams, R.M., Wang, X., Balázsi, G., and Collins, J.J. (2010). Tuning and controlling gene expression noise in synthetic gene networks. Nucleic Acids Res 38, 2712-2726.

Negi, S., Imanishi, M., Matsumoto, M., and Sugiura, Y. (2008). New redesigned zinc-finger proteins: design strategy and its application. Chemistry 14, 3236-3249.

Nomura, W., and Sugiura, Y. (2007). Design and synthesis of artificial zinc finger proteins. Methods Mol Biol 352, 83-93.

Park, S.H., Zarrinpar, A., and Lim, W.A. (2003). Rewiring MAP kinase pathways using alternative scaffold assembly mechanisms. Science 299, 1061-1064.

Paulsen, C.E., and Carroll, K.S. (2009). Chemical dissection of an essential redox switch in yeast. Chem Biol 16, 217-225.

Peisajovich, S.G., Garbarino, J.E., Wei, P., and Lim, W.A. (2010). Rapid diversification of cell signaling phenotypes by modular domain recombination. Science 328, 368-372.

Pomposiello, P.J., and Demple, B. (2001). Redox-operated genetic switches: the SoxR and OxyR transcription factors. Trends Biotechnol 19, 109-114.

Pósfai, G., Plunkett, G. 3rd, Fehér, T., Frisch, D., Keil, G.M., Umenhoffer, K., Kolisnychenko, V., Stahl, B., Sharma, S.S., de Arruda, M., et al. (2006). Emergent properties of reduced-genome Escherichia coli. Science 312, 1044-1046.

Radhika, V., Proikas-Cezanne, T., Jayaraman, M., Onesime, D., Ha, J.H., and Dhanasekaran, D.N. (2007). Chemical sensing of DNT by engineered olfactory yeast strain. Nat Chem Biol 3, 325-330.
Ro, D.K., Paradise, E.M., Ouellet, M., Fisher, K.J., Newman, K.L., Ndungu, J.M., Ho, K.A., Eachus, R.A., Ham, T.S., Kirby, J., et al. (2006). Production of the antimalarial drug precursor artemisinic acid in engineered yeast. Nature 440, 940-943.

Saito, H., Kobayashi, T., Hara, T., Fujita, Y., Hayashi, K., Furushima, R., and Inoue, T. (2010). Synthetic translational regulation by an L7Ae-kink-turn RNP switch. Nat Chem Biol 6, 71-78.

Sera, T. (2010). Generation of cell-permeable artificial zinc finger protein variants. Methods Mol Biol 649, 91-96.

Shen, N., Ko, J.H., Xiao, G., Wesolowski, D., Shan, G., Geller, B., Izadjoo, M., and Altman, S. (2009). Inactivation of expression of several genes in a variety of bacterial species by EGS technology. Proc Natl Acad Sci U S A 106, 8163-8168.

Silverman, J., Liu, Q., Lu, Q., Bakker, A., To, W., Duguay, A., Alba, B. M., Smith, R., Rivas, A., Li, P., et al. (2005). Multivalent avimer proteins evolved by exon shuffling of a family of human receptor domains. Nat Biotechnol 23, 1556-1561.

Siolas, D., Lerner, C., Burchard, J., Ge, W., Linsley, P.S., Paddison, P. J., Hannon, G.J., and Cleary, M.A. (2005). Synthetic shRNAs as potent RNAi triggers. Nat Biotechnol 23, 227-231.

Smith, H.O., Hutchison, C.A. 3rd, Pfannkoch, C., and Venter, J.C. (2003). Generating a synthetic genome by whole genome assembly: phiX174 bacteriophage from synthetic oligonucleotides. Proc Natl Acad Sci U S A 100, 15440-15445.

Sprengel, R., and Hasan, M.T. (2007). Tetracycline-controlled genetic switches. Handb Exp Pharmacol, 49-72.

Steen, E.J., Kang, Y., Bokinsky, G., Hu, Z., Schirmer, A., McClure, A., Del Cardayre, S.B., and Keasling, J.D. (2010). Microbial production of fatty-acid-derived fuels and chemicals from plant biomass. Nature 463, 559-562.

Stricker, J., Cookson, S., Bennett, M.R., Mather, W.H., Tsimring, L.S., and Hasty, J. (2008). A fast, robust and tunable synthetic gene oscillator. Nature 456, 516-519.

Suess, B., Fink, B., Berens, C., Stentz, R., and Hillen, W. (2004). A theophylline responsive riboswitch based on helix slipping controls gene expression in vivo. Nucleic Acids Res 32, 1610-1614.

Suryawanshi, H., Scaria, V., and Maiti, S. (2010). Modulation of microRNA function by synthetic ribozymes. Mol Biosyst 6 , 1807-1809.

Tietze, L.F., Düfert, A., Lotz, F., Sölter, L., Oum, K., Lenzer, T., Beck, T., and Herbst-Irmer, R. (2009). Synthesis of chiroptical molecular switches by pd-catalyzed domino reactions. J Am Chem Soc 131, 17879-17884.

Tigges, M., Marquez-Lago, T.T., Stelling, J., and Fussenegger, M. (2009). A tunable synthetic mammalian oscillator. Nature 457, 309-312.

Winkler, W.C. (2005). Riboswitches and the role of noncoding RNAs in bacterial metabolic control. Curr Opin Chem Biol 9, 594-602.

Yen, L., Svendsen, J., Lee, J.S., Gray, J.T., Magnier, M., Baba, T., D'Amato, R.J., and Mulligan, R.C. (2004). Exogenous control of mammalian gene expression through modulation of RNA selfcleavage. Nature 431, 471-476.

Zhang, K., Sawaya, M.R., Eisenberg, D.S., and Liao, J.C. (2008). Expanding metabolism for biosynthesis of nonnatural alcohols. Proc Natl Acad Sci U S A 105, 20653-20658.

Zhang, X., Schaffitzel, C., Ban, N., and Shan, S.O. (2009). Multiple conformational switches in a GTPase complex control co-translational protein targeting. Proc Natl Acad Sci U S A 106, 1754-1759. 